Cinémas

Revue d'études cinématographiques

Journal of Film Studies

\title{
Présentation. À quoi bon des poètes ...
}

\section{Silvestra Mariniello}

Volume 27, numéro 1, automne 2016

Pasolini, cinéaste civil

URI : https://id.erudit.org/iderudit/1041105ar

DOI : https://doi.org/10.7202/1041105ar

Aller au sommaire du numéro

Éditeur(s)

Cinémas

ISSN

1181-6945 (imprimé)

1705-6500 (numérique)

Découvrir la revue

Citer ce document

Mariniello, S. (2016). Présentation. À quoi bon des poètes .... Cinémas, 27(1),

7-20. https://doi.org/10.7202/1041105ar d'utilisation que vous pouvez consulter en ligne.

https://apropos.erudit.org/fr/usagers/politique-dutilisation/ 


\section{Présentation. À quoi bon des poètes ${ }^{1}$...}

\section{Silvestra Mariniello}

La divine mimesis, écrite entre 1963 et 1965, publiée inachevée en 1975 comme "document", selon la volonté de Pasolini lui-même, est l'un des textes les plus importants pour réfléchir à la rupture entre les années 1950 et les années 1960, et pour aborder la crise qui amène Pasolini à passer de la littérature au cinéma et à s'intéresser au "tiers-monde». La divine mimesis serait le document de cette crise et de ce passage entre deux époques. Un texte difficile, complexe comme toutes les allégories, mais fondamental, parce qu'il évoque les conséquences profondes et dévastatrices de l'émergence de la société de consommation et proclame l'urgence de trouver de nouvelles possibilités, de nouvelles formes d'engagement.

Le projet de reprendre Dante et son voyage en enfer n'était pas nouveau pour Pasolini, pas plus qu'il n'arrive à son terme avec La divine mimesis: c'est un projet qui, au fil des années, prend différentes formes, qu'il s'agisse des "fragments» de $L a$ mortaccia, en 1959, ou d'une ouvre comme Pétrole, le roman posthume (publié en 1992). La divine mimesis est en prose mais, comme La divine comédie, elle est divisée en chants. Dans les deux premiers chants, les seuls qui sont achevés, Pasolini est fidèle au texte de Dante presque à la lettre. Comme Dante avant lui, Pasolini (1975, p. 9), "vers quarante ans", se trouve «à un moment très obscur» de sa vie. Le poète contemporain, comme jadis le poète médiéval, est secouru et guidé à travers les difficultés du voyage par quelqu'un: pour Pasolini il ne s'agit pas de Virgile, mais d'une "figure [...] jaunie par le silence" (p. 18) dans laquelle le poète devra se reconnaître: il s'agit du Pasolini poète civil, du Pasolini des années 1950. C’est lui qui conduira le "nouveau poète» dans le monde: "Outre, toi et moi, nous n'irons pas parce que le monde finit avec le monde" (p. 25). 
Dans les deux premiers chants, Pasolini se distancie des années 1940-1950 et du Parti communiste italien, donc d'une forme d'engagement, et se met en route vers quelque chose de nouveau. Le voyage commence par une cérémonie au cinéma Splendid, théâtre d'une réunion du Parti, où l'obscurité laisse la place à une lumière, la lumière de la "vieille vérité", qui n'est qu'une autre forme d'obscurité. Dans ces pages, Pasolini dénonce la séparation du Parti — dont la conception politique est désormais inadéquate à la réalité historique et économique de l'Italie et de l'Europe des années 1960 - d'avec le monde. Les principales limites du marxisme, et Pasolini le répète à plusieurs reprises pendant ces années, sont le rationalisme et l'abstraction. La voie «juste» (p. 14), seulement rationnelle, ne peut que mener à l'obscurité; pour en sortir, il faut affronter de nouveaux problèmes, trouver de nouvelles réponses.

Comme Dante, Pasolini se repose un peu sur son chemin avant de se retrouver face aux trois fauves, la panthère, le lion et la louve, allégories des vices humains, mais surtout incarnations de ses tendances profondes qui le confrontent à sa propre obscurité. Son texte, qui se détache de celui de Dante d'une façon très intéressante, analyse en une série d'actions négatives ce moment d'arrêt :

Je me reposai un peu, je ne pensai pas, je ne vécus pas, je n'écrivis pas: comme un malade; puis je me remis en marche (c'est la vieille histoire). Montant la pente déserte, où vraiment je pouvais dire que j'étais seul (Pasolini 1975, p. 14).

Le poète s'arrête, paralysé par l'obscurité à laquelle la voie "juste» l'a conduit; il n'écrit pas, ne pense pas, ne vit pas, puis il recommence à avancer, et cette fois il est tout seul, parce qu'il est le seul à accepter les conséquences de l'abandon de cette voie "juste». Ce voyage qu'il a entrepris doit l'amener à comprendre son travail d'écrivain et son rapport à la réalité d'une autre façon. Le cinéma sera un pas dans cette direction, vers une abolition de la distance entre le langage et le monde.

Si la panthère incarne l'attachement à la vie filtré par l'amour maternel et les valeurs chrétiennes — «ne bougeait pas devant mes yeux comme une mère-garçon, comme une église-garçon» 
(p. 15) - et que la peur de perdre cet amour et ces valeurs, objets "d'une affection terrible» (ibid.), le repousse vers le fond de la vallée, le lion incarne le poète arrogant qui, par son «savoir dévorer»(p. 16), détruit la réalité et effraye le protagoniste conscient de la difficulté de "s'empêcher de faire usage» (ibid.) de cette science dévoratrice. Mais des trois fauves, la louve, allégorie du désir sexuel, est la plus dangereuse. Elle fait peur non pas «par ce qu'elle représente de dégradant» (p. 18), mais par sa façon de s'imposer à la conscience: elle ne se laisse pas ignorer, refouler, elle est une "présence indiscutable» (ibid.). Pasolini se met en marche vers le sommet de la colline, "pris par une nouvelle forme de vitalité» (ibid.), rendue possible par «le manque de tout" (ibid.), par le vide qu'il a laissé derrière, et qui lui permet d'avancer jusqu'à ce que la louve s'interpose pour l'empêcher d'aller plus loin. La louve est le désir sexuel, mais le désir sexuel devenu obsession, déformé par la société de l'époque, rendu monstrueux par l'ostracisme et la marchandisation. Au vers de Dante "elle me repoussait là où le soleil se tait ${ }^{2}$ " - où l'image évoque la vallée sombre et, indirectement, la situation historique contemporaine au poète - correspond l'allusion pasolinienne au silence de qui ne remet pas en question la "vieille vérité»:

J'étais rejeté en arrière par la tentation de m'en retourner là où il n'est demandé au fond que de se taire (Pasolini 1975, p. 18).

Taire sa propre sexualité? Sa propre différence? Taire le corps et ses besoins? Taire la passion pour ne laisser de place qu'à l'idéologie comme la voie "juste» l'exige?

C'est à ce moment que Pasolini rencontre son guide et que celui-ci, comme jadis Virgile à Dante, lui propose de changer de route:

Et tandis que je m'effondrais, rendu justement ridicule par mon ancienne victoire sur un monde auquel $j$ 'appartenais sans aucune raison de me retenir plus haut, désormais privé de l'autorité de la poésie, et rendu ignorant par les longues fréquentations obscurantistes, concrètes, pratiques et mystiques, voici que m'apparut une figure, dans laquelle je devais encore une fois me reconnaître, jaunie par le silence (Pasolini 1975, p. 18). 
L'ancienne victoire à laquelle Pasolini fait allusion est peutêtre celle d'une forme de connaissance et d'écriture par laquelle le poète "contrôlait" le monde et qui n'est plus possible. On peut lire dans ce passage une remise en question de la forme de connaissance et d'action que la poésie civile incarnait, en faveur d'une autre forme de connaissance (et d'action) qui, elle, serait immanente. Le poète civil possédait une connaissance qui transcendait le monde; il occupait, comme le lion, une position de supériorité, d'où il pouvait observer et connaître pour "dévorer", pour s'approprier des choses, pour se représenter son appartenance au monde et l'action à y accomplir. Être privé de l'autorité de la poésie civile signifie se précipiter dans le monde pour le connaître autrement et devoir repenser l'action (l'engagement) du poète. Le cinéma, Pasolini ne se lassera pas de le répéter, permet d'exprimer la réalité par la réalité et de ne jamais en sortir. En 1963, Pasolini a déjà réalisé Accattone (1961), Mamma Roma (1962), La rabbia (1963) et La ricotta (1963). Le cinéma lui révèle — et lui permet d'exprimer — son amour désespéré pour la réalité.

Pasolini met en scène dans ces pages de La divine mimesis la fin d'une époque, d'une façon de concevoir l'engagement et la poésie. Le guide, comme Virgile chez Dante, raconte ses origines, et le lecteur apprend qu'il s'agit de nul autre que de Pasolini lui-même, mais dans les années 1950.

Je fus poète - ajouta-t-il, rapide, comme s'il voulait maintenant dicter son épitaphe - , je chantais la division de la conscience de celui qui a fui sa ville détruite et s'en va vers une ville qui est encore à construire. Et qui, dans la douleur de la destruction mêlée à l'espérance de la fondation, accomplit obscurément son mandat... (Pasolini 1975, p. 20.)

Le poète civil chantait des thèmes civils comme la division de la conscience, la destruction due à la guerre, la reconstruction. Mais plutôt que de chanter le progrès, la fondation d'un nouveau monde, comme "la vieille vérité » l'aurait exigé, il chantait le déchirement entre l'ancien qui est fini, qui a été détruit, et le nouveau qui n'a pas encore été reconstruit. Dans la dénonciation de ce déchirement, son mandat, qui lui imposait de chanter 
le nouveau, d'ignorer le gouffre entre la destruction et la reconstruction, entre la passion et l'idéologie, le désir et la raison, s'était épuisé. "La blessure d'un doute, la douleur d'un déchirement deviennent rapidement des maux privés dont les autres ont raison de se désintéresser» (p. 21). À ce guide qui, malgré tout, est le plus apte à protéger le poète contre la louve qui le menace, Pasolini confie sa crainte que le fauve ne lui ôte la force et la volonté de s'exprimer. Au début du chant, Pasolini parle "des temps merveilleux [...] où dans chacun de [s]es actes qu'il fût arbitraire, puéril ou coupable — il était clair qu ['il] faisai[t] l'expérience d'une forme de vie à seule fin de l'exprimer" (p. 10). La louve qui le menace ne menace pas, comme chez Dante, sa vie, mais sa capacité de s'exprimer et peut-être de faire l'expérience d'une forme de vie. À cela le poète civil répond qu' «il faut changer de route [...] si une situation paraît dangereuse ou indigne» (p. 23). L'idée de changer de route renvoie ici également à l'écriture: prendre un autre chemin signifie écrire autrement ou, peut-être, passer de la littérature au cinéma. Le désir sexuel serait devenu obsession et l'obsession aurait vidé le désir de sens et de réalité. Vidé de sens, le désir devient inexprimable. Le cinéma prendrait-il la relève pour dire autrement l'expérience du désir, de l'amour de la réalité, du sentiment religieux, jusqu'à l'expérience de l'enfer petit-bourgeois? Théorème (Teorema, 1968), Porcherie (Porcile, 1969), les films de la Trilogie de la vie - Le Décaméron (Il Decameron, 1971), Les contes de Canterbury (I racconti di Canterbury, 1972), Les mille et une nuits (Il fiore delle mille e una notte, 1974) — et Salò (1975) répondront à la menace de la louve, chacun en développant un aspect différent de la réflexion sur le désir, la sexualité et le pouvoir, selon l'époque à laquelle ils appartiennent.

C'est dans ces pages de La divine mimesis que Pasolini, en reprenant la prophétie dantesque du lévrier, annonce l'ère du capital multinational:

Celui-là, si bien monté, ce ne sera pas un patron d'usines ou de chaînes de journaux, il ne possédera pas de domaines dans le Sud, mais ses richesses seront l'esprit d'entreprise, le capital monétaire et la patrie multinationale (Pasolini 1975, p. 25). 
C'est la naissance d'un ordre complètement différent de celui qui était issu de l'union entre la religion instituée et le capital national: un ordre indifférent à la mort de tant de jeunes cubains ou algériens, de Reggio ou de Palerme, grecs ou espagnols. Le sens de la rupture et du passage des années 1950 aux années 1960 devient explicite. L'avènement du capital monétaire multinational tue le Pouvoir traditionnel, incluant l'Église; il rend la lutte des classes, incarnée dans le sacrifice de tant de jeunes camarades, vaine; il menace, comme la louve, l'activité de l'écrivain, sa capacité à s'exprimer, et c'est là que l'écrivain doit changer de route.

Dans le texte de Pasolini, l'allégorie des trois fauves réécrit la temporalité hors de la continuité que l'idée de progrès présuppose. D'un côté, les fauves renvoient à la société contemporaine, mais dans son hétérogénéité, dans sa tension entre le vieux et le nouveau, entre le privé et le public; de l'autre, par la référence à Dante, ils renvoient à un moment historique passé: le présent des années 1960 se lie au Moyen Âge. La valeur du temps est celle de la différence, de la discontinuité. Discontinuité entre le temps de Dante et celui de Pasolini, entre le temps des fauves et celui du capital monétaire, entre le temps où l'intellectuel avait un mandat et celui où il n'en a plus. Le langage, pour reprendre les mots de Paul de Man (1971, p. 207), «s'établit dans le gouffre de cette différence temporelle».

Le gouffre de la différence temporelle comme lieu de l'action se définit ultérieurement et se concrétise à plusieurs reprises dans le deuxième chant: d'abord quand Pasolini demande à la «vieille inspiration" de l'aider dans sa nouvelle mission, au moment où son guide, justement, le petit poète civil des années 1950, évoque par son physique - ses mouvements, ses vêtements, son corps - une réalité historique provinciale passée qui coexiste avec la nouvelle, comme la double personne du poète (à la fois guide et voyageur) le suggère; et ensuite quand le poète est confronté à la langue: une langue homologuée, sans plus de différences entre la langue cultivée et la langue vulgaire, médium d'une autre connaissance, d'un autre rapport à la réalité: 
Aide-moi, toi, dans cette trahison: dans ce qui est la pire des fantaisies visionnaires où tu m'as tant de fois assisté. Aide-moi à donner corps aux abstractions qui veulent être comme neuves, avec la vieille consistance de l'imagination domestique et élégiaque!

Dans ce passage, Pasolini (1975, p. 29) met en scène le drame de l'écrivain contemporain, placé entre la vieille inspiration qui produit une vision de la réalité désormais inadéquate - une vision totalisante, panoramique, ordonnatrice - et quelque chose de nouveau qui ne se laisse pas dire. La force de ce passage réside surtout dans l'ouverture d'un espace d'action, dans la suggestion d'une dynamique entre l'ancien et le nouveau - une dynamique distincte de la dialectique matérialiste projetée, elle, vers la synthèse idéale des différences. Le texte de Pasolini insiste sur la matérialité du rapport entre le passé et le présent, entre l'histoire et la préhistoire; ce rapport est la poésie et est aussi le cinéma. Le texte se concentre sur la différence, sur le contraste entre les deux moments, sur le gouffre qui les sépare, plutôt que sur la plénitude d'une solution. Le poète invoque, même si elle ne sert plus à rien, la vieille inspiration, dont l'inutilité renforce le sentiment du gouffre, un gouffre ouvert par la tentative vaine, mais inévitable, d'y avoir recours. Dans ce vide se produit «la pire des fantaisies visionnaires»: la tentative de garder ensemble le passé et le présent, de «donner corps aux abstractions [...] avec la vieille consistance de l'imagination domestique et élégiaque ".

L'image de Pasolini-Virgile qui avance insouciant sous le regard du Pasolini des années 1960 provoque le même sentiment que celui que l'apostrophe à la vieille inspiration avait suscité. En mettant l'accent sur la différence entre les deux réalités historiques incarnées, le texte suggère le mouvement qui à la fois les unit et les sépare: l'une ne devient pas le résultat ni n'est le présupposé de l'autre. Une autre dimension temporelle s'ouvre avec la référence explicite à Dante qui a déjà fait ce même voyage: alors que le rapport au monde passait chez Dante par la médiation d'une langue qui se différenciait en langue "cultivée » et en langue "vulgaire» — et qui permettait, d'un côté, de «s'élever» et de "tout voir de loin», et, de l'autre, de «s'abaisser» et de 
"tout voir de près» —, aujourd'hui, dit Pasolini (1975, p. 3233), il n'y a qu'une langue, la langue de la haine:

[...] la mienne - historiquement mienne [...] celle de mon temps, celle de mon père, de ma mère, de mes professeurs, de mes fournisseurs, de mes journaux, de ma radio, de ma télévision, de mes couilles!

C'est elle qui médiatise le rapport à la réalité, qui produit des formes de connaissance et de compréhension de cette réalité, mais lesquelles? Ce n'est pas la réponse qui intéresse le poète, mais le gouffre qui se crée dans la différence entre la langue de la haine et la langue de Dante, et la possibilité d'action que ce gouffre libère.

Un «désir de faire (dans ce monde inutile)» (p. 34) s'impose comme le thème de ces pages. À la fin du deuxième chant de $L a$ divine mimesis, l'allégorie des fleurs, inspirée par la similitude dantesque, est en fait une philosophie de l'agir, de l'être dans le monde. Le poète se compare à l'une de ces petites fleurs «qui viennent des régions du passé jamais mort du cosmos et qui campent» (p. 36) dans le présent: la possibilité d'action, la vie s'affirment dans cette région de l'esprit et de la parole où «le passé se confond avec le présent, et [où] un pré est à la fois ici et dans le cosmos!» (ibid.) La similitude dantesque évoque l'image de quelqu'un qui se redresse sous l'effet de la lumière, qui retrouve sa force, perdue à cause de la nuit (nuit de la raison et de la foi); Dante, après avoir hésité, retrouve ses certitudes; le courage de traverser l'enfer lui vient de la confiance en la vérité qui l'illumine. La métaphore pasolinienne évoque plutôt les alternances, l'être entre la mort et la vie, et la caducité de l'expérience. L'écart entre les différentes réalités définies et définissables, dans le temps et dans l'espace, devient pour Pasolini le lieu de l'action: le point où changer de route.

L'avènement de la société de consommation, l'homologation des classes (désormais il n'y a que la petite bourgeoisie, les autres ne sont que des survivances) - en d'autres mots, l'enfer de $L a$ divine mimesis - poussent Pasolini à chercher d'autres réalités, à partir, à abandonner cette Europe qui n'est plus la sienne ${ }^{3}$. Les années 1960 sont les années des voyages en Afrique, en Inde et 
au Moyen-Orient, et ce sont aussi les années de la découverte du cinéma comme «langue écrite de la réalité» (Pasolini 1966). Le changement de route se fait dans le sens littéral, géographique - de l'Europe à l'Afrique et à l'Asie —, et dans le sens métaphorique - de la littérature au cinéma. La poésie accompagne et favorise ce changement, en devient l'agent: Pasolini écrit des poèmes pendant ses voyages, et pendant les repérages et les tournages de ses films. Il parle souvent de ses projets cinématographiques comme de poèmes (par exemple dans le cas du titre des Notes pour un poème sur le tiers-monde ou à propos de Porno-Teo-Kolossal). La poésie, comme le cinéma et avec le cinéma, devient le médium de la réécriture de l'espace et du temps, de la géographie et de l'histoire. La poésie partage la matérialité du cinéma (des images, du rythme, des sons) et sa dynamique. La poésie et le cinéma sont des événements, des réalités en devenir, comme la parole orale qui est vivante - à la différence de la parole écrite qui devient chose, et qui se laisse disposer horizontalement sur la page. Changement de route donc, vers d'autres réalités historiques, linguistiques et géographiques. La géographie pasolinienne est une géographie spéciale où «Bandung est la capitale des trois quarts du monde [...] et aussi de la moitié de l'Italie» (Pasolini 1977, p. 145), où la voie Appienne mène à Kochi, où il y a continuité entre la Guinée et la plaine du Pô ou la campagne des Pouilles, et où la Lucanie, qui ressemble au Maroc, est proche du Vietnam. Cette géographie est inséparable d'une conception de l'histoire qui remet en question la linéarité du progrès, ainsi que la possibilité de l'harmonie, et du retour. Dans cette géographie et dans cette temporalité, l'Afrique devient une allégorie; elle ne renvoie pas, comme l'Afrique de Sartre ou de Senghor, à une identité s'exprimant en une série d'équivalences (Afrique $=$ négritude $=$ poésie pure $=$ authenticité $=$ passion $=$ rapport non instrumental avec la nature...), elle renvoie à une différence et à une série d'écarts qui ne pourront pas être comblés. Dans le poème $L a$ Guinée, publié en 1962, la négritude devient une allégorie qui menace la conception dialectique de l'histoire. En reprenant le concept des poètes africains pour le transposer dans un univers «blanc», Pasolini associe la situation des paysans et des 
sous-prolétaires italiens à celle des Africains dans les colonies qui luttent pour leur indépendance. Négritude «blanche» et négritude noire: la nature paradoxale de la première renvoie à la seconde pour créer un nouvel espace d'action. La négritude «blanche» signifie par elle-même la distance et la différence temporelle qui la séparent de son modèle: un élément frappant de La Guinée est que le poète y parle de négritude au retour en Italie — «Ainsi je me réveille, le matin, en Italie [...] La Négritude est dans ces prairies blanches" (Pasolini 1964, p. 13) — plutôt que dans la partie du poème située en Afrique; mais y a-t-il vraiment une partie située en Afrique et une autre en Italie? Est-il vraiment possible de tracer les limites entre les deux réalités? Les deux signes "négritude" ne peuvent pas être identiques: le second renvoie au premier et sa signification consiste précisément en ce renvoi à un signe qui le précède et avec lequel il ne peut pas coïncider. Pasolini ne crée pas une similitude, n'établit pas d'équivalence entre les deux négritudes. Si le discours de $L a$ Guinée visait à établir une identité entre la négritude "blanche» et la négritude africaine, il en résulterait une vision unitaire et totalisante de peuples différents qui se dirigent ensemble vers un futur sans races, unis dans la lutte commune pour le progrès. Mais, dans La Guinée, la négritude "blanche» signifie aussi la différence par rapport à la négritude africaine, l'impossibilité pour les deux de coïncider. Il faut alors chercher d'autres voies que celles du progrès et de la synthèse sartrienne. "La Négritude [...] qui sera raison" est celle qui se constitue dans la différence entre les deux, dans le vide produit par la transformation rapide du monde et donc dans la nécessité de réinventer la façon de l'habiter. L'Afrique de Pasolini invite à repenser l'histoire, et nie la possibilité de la dialectique et du progrès.

Les textes qui composent ce numéro reprennent, à plus de quarante ans de la mort de Pasolini, les grandes questions que La divine mimesis, récit visionnaire, abordait. En fait, cette œuvre inachevée, ce "document», semble être le squelette caché du corps formé par les articles composant ce numéro.

"Pasolini, années 1940-1942: généalogie d'une poétique antifasciste", d'Anne-Violaine Houcke, développe l'idée d'une "poétique de l'invention» qui aurait déjà été au cœur des choix 
artistiques pasoliniens face à la rhétorique fasciste. Une telle poétique consiste en la mise au jour de réalités qui étaient déjà là, mais «exclues du désir du regard». Ces réalités marginales, refoulées, ces débris qui n'avaient pas de place dans le discours architectural, sociologique, littéraire et artistique du fascisme peuplent en particulier les écrits en dialecte frioulan de Pasolini, où la langue devient médiation d'un rapport au réel que le fascisme menaçait: elle est "capable de faire ressurgir une terre paysanne, archaïque» et de rapprocher le ciel de la personne qui le nomme. Dès le début des années 1940, Pasolini s'opposait à la culture officielle soutenue par le régime et cherchait, en s'inspirant de Cézanne, de Freud, d'Ungaretti, de Longhi, de nouvelles voies hors de la rhétorique du régime, déracinée de la réalité des gens. Cet article révèle la continuité et la cohérence de la philosophie de Pasolini, qui voit le passé dans sa capacité à féconder le présent, à "s'y reproduire, non pas à l'identique, mais sous une nouvelle forme» — une philosophie engagée à déconstruire toute idéologie qui nie ou monumentalise le passé.

"Un cinéma blessé : Pasolini et le mythe de la ville intacte», de Marco Bertozzi, s'inscrit dans la continuité de la réflexion d'Anne-Violaine Houcke en se concentrant sur ces réalités invisibles inaccessibles au "désir du regard»: que ce soit la banlieue romaine, avec ses nouveaux logements populaires qui poussent à côté des ruines de la Rome antique, ou qu'il s'agisse des villes de Sanaa et d'Orte, "immolées sur l'autel de la toute-puissante rente foncière" et d'une modernité qui ne respecte pas les traditions. L'ancienne route en pierre qui mène à la porte d'Orte est un humble élément d'une architecture mineure, qui représente la ville entière dans "ses aspects les plus ordinaires et les moins monumentaux». Le cinéma documentaire chez Pasolini réactive le "désir du regard» sur les réalités marginales de l'histoire tout en interrogeant son propre pouvoir. Il devient «l'endroit où les anciennes appartenances morales et identitaires commencent à craquer». L'article de Bertozzi se termine sur une réflexion plus générale à propos d'un cinéma qui continuerait d'entendre le "cri de douleur de Pasolini devant les blessures faites aux villes italiennes historiques ", mais qui s'engagerait sur une nouvelle route, ouverte par l'acceptation de ces 
paysages urbains démembrés en tant que work in progress; un cinéma qui, comme celui de Pasolini, «s'écarte des sentiers battus dans sa rencontre et son combat avec le monde»... mais le monde a changé et la recherche d'autres façons de faire devient urgente.

" "Seuls les marxistes aiment le passé": le tiers-mondisme de Pier Paolo Pasolini dans le genre des appunti», de Luca Caminati, reprend des questions posées par Marco Bertozzi, notamment à propos de Sanaa et d'Orte, toutes deux dévastées par une "modernisation forcée " qui "défigure et désintègre l'humanité tout autant que le paysage». L'Italie et le Yémen font partie du même tiers-monde, associés par cette modernisation forcée. L'auteur qualifie l'engagement de Pasolini pour le tiers-monde d' "orientalisme hérétique" et, en rapprochant Pasolini de Frantz Fanon, propose de le considérer comme l'un des penseurs de la décolonisation. Le tiers-monde est pour Pasolini plus qu'une réalité géographique et politique: il devient une allégorie, un espace libéré "pour la création de nouvelles significations", une solution de rechange au modèle néocapitaliste occidental. En tant qu' "artiste-ethnographe», Pasolini invente une forme - les appunti, «ce genre étrange à la croisée de l'éthique et de l'esthétique» —, qui semble la plus apte à rendre compte des sociétés en devenir dans l'Afrique des années 1960, et une pédagogie qui «ne verse jamais dans le didactisme; au contraire, elle est la démonstration d'un processus, celui du da farsi». Le nouvel engagement du poète, à qui le cinéma révèle la complexité d'une réalité nécessairement contradictoire, est dans cette pédagogie qui est une double "méditation sur un objet d'études (l'autre et l'altérité) et sur le dispositif du film ", sur le langage du cinéma.

"Le Pasolini des derniers temps dans le "maintenant" de sa lisibilité ", d'Hervé Joubert-Laurencin, fait écho à cette nouvelle pédagogie qui «est la démonstration d'un processus, celui du $d a$ farsi », dont parle Luca Caminati, à l'acceptation de "l'inachèvement de ce work in progress rempli de paysages urbains démembrés " qu'évoque Marco Bertozzi et à la pensée performative d'un passé capable de féconder le présent dont il est question dans l'article d'Anne-Violaine Houcke. L'auteur écrit à partir du 
«maintenant» de la France (et du monde) de 2014-2015 et dit: "Salò, c'est maintenant", et encore: "nous vivons ce rêve noir dans notre "maintenant" ». Qu'est-ce qu'une réflexion sur les derniers temps de Pasolini, sur Salò maintenant, peut nous apporter dans la quête éthique et politique qui caractérise notre engagement en des temps si sombres? Pour y répondre, Hervé Joubert-Laurencin passe par une discussion du terme «lisibilité» et de son usage chez le dernier Pasolini. Ce terme qui revient dans l'"Abjuration de la Trilogie de la vie», dans le traité pédagogique Gennariello, ainsi que dans La divine mimesis, renvoie à Roland Barthes et en particulier à son analyse d'une nouvelle de Balzac où il associe lisible à "classique" et scriptible à «moderne». Le lecteur (ou le spectateur) transforme le lisible en scriptible dans la mesure où il reconnaît dans la suspension caractérisant la fin de la nouvelle (ou du film) un acte capable de "faire éclater, à l'intérieur de la représentation classique, toute représentation classique», voire toute représentation. Cette réalité qu'on ne peut plus objectiver pour y intervenir, mais que nous avons à déchiffrer, cette réalité lisible est l'héritage du dernier Pasolini qui nous permet "d'entrer dans l'époque abhorrée en la condamnant sans la refuser", de "travailler dans le "maintenant" " vers des futurs possibles.

"Du développement du capitalisme à l'infini plan-séquence: les quatre "utopies" de Porno-Teo-Kolossal", de Julie Paquette, conclut ce dossier. Le concept de révolution utilisé par l'auteure pour décrire le périple des deux protagonistes du scénario est à entendre ici "au sens du mot latin revolutio, qui signifie "retour", un retour à l'origine - mais une origine troublée", hors de toute téléologie, sans fin. Le passé qui féconde le présent dans l'invention artistique évoqué dans l'article d'ouverture, le devenir urbain des villes démembrées exigeant d'autres formes d'engagement, le da farsi des appunti et "les significations jamais achevées, toujours présentes" de Salò confluent dans cette idée de révolution qui défie le montage-mort et ouvre sur un "a-venir indéfini ". Dans La divine mimesis, le Pasolini poète civil disait au Pasolini des années 1960: "Outre, toi et moi, nous n'irons pas parce que le monde finit avec le monde"; le projet de film Porno-Teo-Kolossal, dans la lecture originale et 
courageuse que Julie Paquette en propose, donne un sens particulier à cette phrase, parce que le monde y est, justement, infini, en transformation constante: "Pasolini abjure, mais ne regrette jamais ce qu'il a fait; il constate que les choses changent et il adapte son engagement en fonction de la manière dont les choses se transforment; et cet engagement n'a qu'un but, lui permettre de mieux connaître cette Terre, dans sa complexité. »

\section{Université de Montréal}

\section{NOTES}

1. Ce texte est en partie une réécriture d'un chapitre de mon livre sur Pasolini, publié aux éditions Cátedra en 1999.

2. Cette citation de La divine comédie est tirée de la traduction de L'enfer par Jacqueline Risset, publiée chez Flammarion en 1992 (p. 27).

3. Voir le poème Israele dans Pasolini 1964 (p. 166).

\section{RÉFÉRENCES BIBLIOGRAPHIQUES}

Man 1971 : Paul de Man, Blindness and Insight: Essays in the Rhetoric of Contemporary Criticism [1971], Minneapolis, Minnesota University Press, 1983.

Mariniello 1999 : Silvestra Mariniello, Pier Paolo Pasolini, Madrid, Cátedra, 1999.

Pasolini 1959 : Pier Paolo Pasolini, La mortaccia (frammenti), dans Ali dagli occhi azzurri, Milan, Garzanti, 1959, p. 243-248.

Pasolini 1964 : Pier Paolo Pasolini, Poesia in forma di rosa [1964], Milan, Garzanti, 1977.

Pasolini 1966 : Pier Paolo Pasolini, «La langue écrite de la réalité» [1966], dans L'expérience hérétique. Langue et cinéma, traduit de l'italien par Anna Rocchi Pullberg, Paris, Payot, 1976, p. 167-196.

Pasolini 1975 : Pier Paolo Pasolini, La divine mimesis [1975], traduit de l'italien par Danièle Sallenave, Paris, Flammarion, 1980.

Pasolini 1977 : Pier Paolo Pasolini, Le belle bandiere, Rome, Editori Riuniti, 1977.

Pasolini 1992 : Pier Paolo Pasolini, Pétrole [1992], traduit de l'italien par René de Ceccatty, Paris, Gallimard, [1995] 2006. 